\title{
Multicast Capacity in MANET with Infrastructure Support
}

\author{
Zhenzhi Qian ${ }^{1,2}$, Xiaohua Tian ${ }^{1}$, Xi Chen ${ }^{1}$, Wentao Huang ${ }^{1}$ and Xinbing Wang ${ }^{1,3}$ \\ 1. Department of Electronic Engineering, Shanghai Jiao Tong University, China \\ 2. State Key Laboratory of Integrated Services Networks, Xidian University, China \\ 3. National Mobile Communications Research Laboratory, Southeast University, China \\ Email: $\{199012315171, x t i a n, q f b z c x, y e l o h u a n g, x w a n g 8\} @ s j t u . e d u . c n$
}

\begin{abstract}
We study the multicast capacity under a network model featuring both node's mobility and infrastructure support. Combinations between mobility and infrastructure, as well as multicast transmission and infrastructure, have already been showed effective ways to increase it. In this work, we jointly consider the impact of the above three factors on network capacity. We assume that $m$ static base stations and $n$ mobile users are placed in an ad hoc network. A general mobility model is adopted, such that each user moves within a bounded distance from its home-point with an arbitrary pattern. In addition, each mobile node serves as a source of multicast transmission, which results in a total number of $n$ multicast transmissions. We focus on the situations in which base stations actually benefit the capacity improvement, and find that multicast capacity in a mobile hybrid network falls into several regimes. For each regime, reachable upper and lower bounds are derived. Our work contains theoretical analysis of multicast capacity in hybrid networks and provides guidelines for the design of real hybrid system combing cellular and ad hoc networks. ${ }^{1}$
\end{abstract}

Index Terms-Wireless ad hoc network; multicast capacity; mobility; infrastructure; hybrid network; scaling law;

\section{INTRODUCTION}

Recent years witness a rapid development in wireless ad hoc networks, in both academic and industrial fields. Kumar and Gupta have showed in their ground breaking work [1] that, even with the optimal scheduling, routing and relaying of packets, the per-node capacity still decreases as $\Theta(1 / \sqrt{n})$ when $\mathrm{n}$ approaches the infinity. Many studies try to improve this disappointing scalability of throughput capacity by introducing different characteristics into ad hoc networks, such as mobility of nodes, an infrastructure of the network: a multicast transmission scheme.

Mobility in ad hoc networks was considered firstly by Tse et al. in [2]. A store-carry-forward relaying scheme was proposed and proven to sustain a $\Theta(1)$ per-node capacity, if each node can visit the whole network area with an uniformly ergodic mobility process. Garetto et

1. The early version of this paper appears in the Proceedings of IEEE Inter-national Conference on Computer Communications(IEEE INFOCOM), 2012. [18] al. generalize the mobility model through a restriction that each moving node is located within a circle of radius $1 / f(n)$ [3]. By mapping the network to a generalized random geometric graph, they have proven that $\Theta(1 / f(n))$ per-node capacity is achievable.

Infrastructure in an ad hoc network provides a more straightforward increase to the capacity. In [4], Liu et al. claim that infrastructure can offer a linear capacity increase in a hybrid network, when the number of base stations increases asymptotically faster than $\sqrt{n}$. In addition, Kozat and Tassiulas [5] prove that if the number of users served by each BS is bounded above, a per-node capacity of $\Theta(1 / \log n)$ can be achieved. In [6], Agarwal and Kumar further extend this result to $\Theta(1)$.

Multicast transmission refers to the transmission from a single node to other $k-1$ nodes, so as to generalize both unicast and broadcast transmissions. In [7], $\mathrm{Li}$ proves that multicast transmission can obtain a per-flow capacity of $\Theta\left(\sqrt{\frac{1}{n \log n}} \cdot \frac{1}{\sqrt{k}}\right)$, which is larger than that of $k$ unicast transmissions. The gain of multicast transmission results from a merge of relay paths within a minimum spanning tree. In [8], Li et al. extend the multicast transmission to a Gaussian channel model and show similar capacity improvement under the corresponding protocol.

Many existing studies focus on the combinations of the above characteristics. Some aim to further increase the network performance, while others try to present a more realistic scenario. In [9] and [10], $\mathrm{Li}$ et al. explore the multicast capacity in a static hybrid network with infrastructure support. Establishing a multicast tree with the help of infrastructure and employing a hybrid routing scheme, they have showed that the achievable multicast capacity in a hybrid network with $m$ BSs is $\Theta\left(\max \left[\min \left(\frac{W_{B} \sqrt{m}}{n_{s} \sqrt{k}}, \frac{W_{c} \sqrt{m}}{n_{s} k}, \frac{W_{a} \sqrt{m}}{n_{s} k}\right), \frac{W_{a}}{n_{s} \sqrt{k}} \cdot \frac{a}{r}\right]\right)$. On the other hand, Huang, Wang et al. study the unicast capacity of mobile hybrid networks in [11], and jointly consider the influences of node's mobility and infrastructure support on it. A per-node capacity is 
$\Theta\left(\frac{1}{f(n)}\right)+\Theta\left(\min \left[\frac{m^{2} c}{n}, \frac{m}{n}\right]\right)$ for strong mobility, and $\Theta\left(\min \left[\frac{m^{2} c}{n}, \frac{m}{n}\right]\right)$ for weak and trivial mobility.

In this paper, we further study the multicast capacity scaling laws of a mobile hybrid network characterizing both mobility and infrastructure. In our model, each of the $n$ users moves around a home-point within a bounded radius. $m$ wire-connected base stations are placed in a wireless ad hoc network, of which the area scales with $\mathrm{n}$ as $f^{2}(n)$. There are totally $n_{c}$ clusters with radius $r=\Theta\left(n^{-R}\right)$ and the number of destinations in the multicast scheme is assumed as $k$. A multicast path can be generated with an infrastructure routing and a pure ad hoc routing, as well as a combination of both. Intuitively, in our hybrid routing scheme, we hope to circumvent the bottleneck of backbone transmission or wireless access for cellular networks and take the advantage of them, thus the capacity can be improved.

Our main contributions: In this paper, we consider the effects of mobility and infrastructure in multicast capacity of a wireless mobile ad hoc network. We divide mobility into three regimes, and present reachable upper bounds and lower bounds for each regime. We assume that bandwidth is $W$ for wireless channel, and $W_{B}$ for wired connections. In cellular routing, we further divide wireless frequency resource $W$ into uplink bandwidth $W_{A}$ and downlink bandwidth $W_{C}$.

- For the first regime (strong mobility regime) where $f(n) \sqrt{\gamma(n)}=o(1),\left(\gamma(n)=\frac{\log n_{c}}{n_{c}}\right)$, the per-node capacity by ad hoc routing is:

$$
\Theta\left(\frac{W}{\sqrt{k} f(n)}\right)
$$

And per-node capacity by cellular routing is:

$$
\Theta\left(\min \left[\frac{m W_{A}}{n}, \frac{m^{2} W_{B}}{k n}, \frac{m W_{C}}{k n}\right]\right)
$$

By always choosing a better routing, the per-node capacity of hybrid routing scheme is:

$$
\Theta\left\{\max \left[\frac{W}{\sqrt{k} f(n)}, \min \left(\frac{m W_{A}}{n}, \frac{m^{2} W_{B}}{k n}, \frac{m W_{C}}{k n}\right)\right]\right\}
$$

- The second regime (weak mobility regime) stand$\mathrm{s}$ for the situation that $f(n) \sqrt{\gamma(n)}=\omega(1)$ and $f(n) \sqrt{\tilde{\gamma}(n)}=o(1)$, where $\sqrt{\tilde{\gamma}(n)}=\frac{1}{r} \sqrt{\frac{\log \left(n / n_{c}\right)}{n / n_{c}}}$. We prove that, in this regime, mobility is only helpful in delivering intra-cluster message. The inter-cluster message can only be transmitted through cellular routing. As a result, the optimal routing scheme is a serial connection of ad hoc routing (phase 1) and cellular routing (phase 2). We prove that the result in the first regime can be mapped to phase 1 here. Consequently, the per-node capacity by ad hoc routing is:

$$
\Theta\left(\frac{W \sqrt{n_{c}}}{n^{R} \sqrt{k}}\right)
$$

Furthermore, the per-node capacity by cellular routing is:

$$
\Theta\left[\min \left(\frac{m W_{A}}{n}, \frac{n_{c} m^{2} W_{B}}{\left(n_{c}-1\right) n k}, \frac{m n_{c} W_{C}}{n k}\right)\right]
$$

Considering the nature of serial connection, the pernode capacity by hybrid routing scheme is:

$$
\Theta\left[\min \left(\frac{W \sqrt{n_{c}}}{n^{R} \sqrt{k}}, \frac{m W_{A}}{n}, \frac{n_{c} m^{2} W_{B}}{\left(n_{c}-1\right) n k}, \frac{m n_{c} W_{C}}{n k}\right)\right]
$$

- The third regime (trivial mobility regime) corresponds to $f(n) \sqrt{\tilde{\gamma}(n)}=\omega(1)$. In this regime, we can prove that mobility is trivial and the network acts as a static one. Previous conclusions in static hybrid networks, such as [4], [9], and [10], can be applied in this trivial mobility regime.

Different from previous studies on hybrid networks [4]- [6], [9], [10], our work takes node's mobility into account. Besides, our work is the first one to consider the effect of a general mobility on multicast transmission. Furthermore, we study multicast capacity in a more realistic network model featuring both mobility and infrastructure support. As a result, our work generalizes both unicast and broadcast capacity results in MANETs and hybrid networks.

Comparing to the conference paper, we add some proofs and remarks in this paper. It is worth noticing that these are significant for providing a better understanding of the difference between MS-MS link capacity and MSBS link capacity, optimal ad hoc routing in uniformly dense networks, the derivation of multicast capacity lower bound, etc. Also, these proofs and remarks offer useful insights in the related topic, such as why we divide mobility into three regimes, the reason why scheduling $\mathrm{S}^{*}$ is optimal, which makes the theoretical analysis smoother and more rigorous.

This paper is organized as follows. We first introduce our network models and assumptions in Section II. Section III and Section IV define the uniformly dense networks, and derive multicast capacity in such networks under ad hoc routing and cellular routing, respectively. In Section V, we combine the results of previous two sections and derive multicast capacity under hybrid routing scheme. Capacity analysis of non-uniformly dense networks is carried out in Section VI. We discuss the proposed multicast routing strategy and protocol designs in Section VII. At last, we conclude our work in Section VIII.

\section{Models AND Assumptions}

We consider a wireless network consisted of $n$ mobile users (also referred as mobile stations, MSs) moving over a bidimensional surface. Communications are carried out in wireless channels with bandwidth $W$ by ad hoc routing, or by cellular routing with the help of $m=\Theta\left(n^{M}\right)$ base stations (BSs), which are connected to each other by optical fibers with bandwidth $W_{B}$. 
We use $X_{i}(t)$ to denote the position of $i$ th MS at any given time $t$, and $Y_{i}(t)$ for $i$ th BS. Since BSs are statically placed in the network, we have $\forall t, Y_{i}(t) \equiv Y_{i}(0) \triangleq Y_{i}$. When referring to both MSs and BSs, we adopt $Z_{i}(t), 1 \leq$ $i \leq n+m$ to denote their locations. $X_{i}^{h}$ is used to denote the location of home-point for $i$ th MS. We further define operator $\|\cdot\|$ as the distance between two points: $d_{i j}=$ $\left\|Z_{i}-Z_{j}\right\|$.

\subsection{Mobility Model}

Definition 1: (Network Extension) $\mathcal{O}$ is a Torus, or a square with wrap-around conditions. The side length of the network scales with $n$ according to a non-decreasing function $f(n)=n^{\alpha}$, where $\alpha \in[0,1 / 2]$. We normalize the whole network to a unit Torus for convenience. Correspondingly, any value representing a distance in the network should be scaled down by $1 / f(n)$.

Remark 1: $\alpha=0$ corresponds to the dense network [1], of which the size remains constant while node density increases linearly with $n$. And $\alpha=1 / 2$ represents the extended network [12], in which network size increase linearly with $n$ while node density remains constant.

It is worth pointing out that ignoring the edge effect of a network is reasonable for avoiding tedious derivations, and is adopted in previous studies [3], [11]. At the same time, normalization of the network area is commonly used for convenience [1], [2]. The above assumptions will not influence the main results in this paper.

Definition 2: (Home-Point Cluster Model) We assume there are $n_{c}=\Theta\left(n^{C}\right)$ clusters with radius $r=\Theta\left(n^{-R}\right)$. All the clusters are independently and uniformly distributed in network $\mathcal{O}$. Then each of the $n$ home-points is randomly assigned to a cluster and placed in it uniformly and independently.

Definition 3: (MS Mobility) We assume that $\left\{X_{i}(t)\right\}$ are independent, stationary, ergodic and rotationinvariant processes with stationary distribution $\phi_{i}(X)$ :

$$
\phi_{i}(X)=\phi_{i}\left(X-X_{i}^{h}\right)=\frac{s\left(\left\|X-X_{i}^{h}\right\|\right)}{\int_{\mathcal{O}} s\left(\left\|X-X_{i}^{h}\right\|\right) d X}
$$

, where $s(d)$ is an arbitrary non-decreasing function with finite range.

Remark 2: $s(d)$ describes MS's presence around its home-point before network normalization. Since

$$
\int_{\mathcal{O}} s\left(\left\|X-X_{i}^{h}\right\|\right) d X \sim 1 / f^{2}(n)
$$

we have:

$$
\phi_{i}(X) \sim f^{2}(n) s\left(\left\|X-X_{i}^{h}\right\|\right)
$$

Remark 3: Our mobility model is similar to that of [3] and [11]. In order to characterize the non-uniform distribution and limited motion observed in the real mobility (see [13], etc.), home-points and $s(d)$ are introduced in our work. Such model can better demonstrate the preferential attachment phenomena in real networks. Furthermore, our mobility and cluster model can generalize other classical mobility models, such as i.i.d. mobility models [12], hybrid random walk models [14] and Brownian motion models [15], by setting $n^{C}=n$ and $f(n)=\Theta(1)$.

Definition 4: (Mobility Regimes) Let $\gamma(n)=\frac{\log n_{c}}{n_{c}}$ and $\tilde{\gamma}(n)=\frac{1}{r^{2}} \log \frac{\left(n / n_{c}\right)}{n / n_{c}}$. We say that MS's mobility is strong if $f(n) \sqrt{\gamma(n)}=o(1)$. Weak mobility corresponds to $f(n) \sqrt{\gamma(n)}=\omega(1)$ and $f(n) \sqrt{\tilde{\gamma}(n)}=o(1)$, while trivial mobility corresponds to $f(n) \sqrt{\tilde{\gamma}(n)}=\omega(1)$

Remark 4: With strong mobility, MSs can exist on cluster boundaries and meet those in other clusters. Under weak mobility, MSs only move within their own clusters, and mobility only helps in delivering intracluster message. In the case of trivial mobility, mobility provides little help in forwarding packets, and the whole network acts like a static one. Also, the mobility regime is an attribute of network, rather than that of MSs. The determinants of mobility regimes are growing speed of network size and clustering level of home-points.

Instead of regularly BS placing method in [4], [9] and [10], the distribution of BSs in this paper matches that of MSs, so as to achieve better utilization of BSs. For $j$ th BS, we randomly select a point $Q_{j}$ according to the cluster model, then uniformly and independently placed the BS in this cluster with distribution $\phi\left(Y-Q_{j}\right)$.

In this paper, we focus on the case that clusters do not overlap with each other w.h.p., which is guaranteed by $M<2 R$. Also, we assume $0 \leq R \leq \alpha$, so that clusters will not shrink with increasing $n$. In order to ensure that every cluster is served by BSs w.h.p., i.e. $m=\omega\left(n_{c}\right)$. An example network under cluster and mobility models is showed in 1.1 in supplementary file.

\subsection{Communication and Interference Models}

Base stations communicate with each other through optical fiber with bandwidth $W_{B}$. This kind of communication will not cause interference to themselves or wireless communications. We assume that the available bandwidth in all the wireless channels is $W$. In ad hoc routing, transmissions fully occupy the wireless bandwidth $W$. In cellular routing, bandwidth is further divided into uplink bandwidth $W_{A}$ and downlink bandwidth $W_{C}$. All the communications in wireless channels are characterized by Protocol Model, which is defined as followed.

Definition 5: (Protocol Model) Both BSs and MSs adopt the same transmission range $R_{T}$ (correspondingly, same transmission power). At each time slot $t$, a wireless transmission from node $i$ to node $j$ is successful only if: 1)

$$
\left\|Z_{i}(t)-Z_{j}(t)\right\| \leq R_{T}
$$

and 2)For any other node $l$ that transmits at $t$,

$$
\left\|Z_{l}(t)-Z_{j}(t)\right\| \geq(1+\Delta) R_{T}
$$

, where $\Delta$ is a constant defining protection zone.

We assume all of the $n$ MSs are source nodes, each of which randomly selects other $k$ MSs as destinations 
and transmits the same data to them. The selection of destinations is well-designed, such that every MS is the destination of other $k$ MSs. Correspondingly, We define the Feasible Throughput and Asymptotic Multicast Per-node Capacity $\lambda$ similar to previous works, such as [1], [7], etc..

\section{Multicast CAPACITY IN UNIFORMLY Dense Networks by Ad Hoc Routing}

In this section, we firstly provide a definition of an uniformly dense network, as well as some characteristics in such network. We show that when a network falls into strong mobility regime, it is equivalent to classify it as an uniformly dense network. Then reachable upper and lower bounds are presented in both pure ad hoc routing and cellular routing for uniformly dense networks. For pure ad hoc routing, we map the mobile network into a random geometric graph, and derive reachable capacity bounds. For cellular routing, we divide the routing scheme into three phases and achieve reachable upper and lower bounds in each phase, as well.

\subsection{Preliminary for Uniformly Dense Networks}

Definition 6: (Local Density) The local density of nodes at any given point $X_{0}$ is defined as:

$$
\rho^{n}\left(X_{0}\right)=\sum_{i=1}^{n} E\left[\mathbf{1}_{X_{i}^{(n)} \in B\left(X_{0}, 1 / \sqrt{n}\right)} \mid \mathbf{H}_{\mathbf{n}+\mathbf{m}}\right]
$$

,where $B\left(X_{0}, 1 / \sqrt{n}\right)$ is the disk centering at $X_{0}$ with radius $1 / \sqrt{n} . \mathbf{H}_{\mathbf{n}+\mathbf{m}}=\left\{Z_{i}^{h}\right\}_{i=1}^{n+m}$ defines the Borelfield of home-points. $E[\cdot]$ stands for expectation, and $\mathbf{1}_{x}$ represents the indicator function.

Definition 7: (Uniformly Dense Networks) We say a network is uniformly dense if for any $X \in \mathcal{O}$, there exist two positive constant $q$ and $Q$, such that

$$
q<\rho^{n}\left(X_{0}\right)<Q
$$

Lemma 1: Suppose that $Z_{i}^{h}$ are placed on $\mathcal{O}$ according to cluster model. Let $\mathcal{A}$ be any given regular tessellation of $\mathcal{O}$, with area $|\mathcal{A}| \geq(16+\delta) \gamma(n)$, for some small $\delta>0$. Let $N(\mathcal{A})$ be the number of home-points of both BSs and MSs inside $\mathcal{A}$. Then it holds w.h.p. :

$$
\frac{n|\mathcal{A}|}{2} \leq \inf N(\mathcal{A}) \leq \sup N(\mathcal{A}) \leq 2 n|\mathcal{A}| .
$$

Lemma 2: If $f(n) \sqrt{\gamma(n)}=o(1)$ and $m=O(n)$, where $\gamma(n)=\frac{\log \left(n_{c}\right)}{n_{c}}$, then the network is uniformly dense.

The proof of Lemma 1 and Lemma 2 are available in both [11] and [16].

Remark 5: $f(n) \sqrt{\gamma(n)}=o(1)$ indicates that nodes can move outside their the home-point clusters, and may move into other clusters. In this sense, mobility plays a fundamental role in exchanging information. Otherwise, nodes cannot exit their cluster and communicate with those in other clusters. Therefore, clustering becomes a obstacle of connectivity.
Definition 8: (Link Capacity) Link capacity between node $i$ and $j$ is defined by the maximal long term data flow between them:

$$
\mu^{S}(i, j)=E\left[\mathbf{1}_{(i, j) \in \pi^{S}(t)} \mid \mathbf{H}_{n+m}\right]
$$

,where $S$ is any given scheduling under protocol model, and $\pi^{S}(t)$ denotes the set of transmission pairs scheduled by $S$.

\subsection{Upper Bound in Uniformly Dense Networks by Ad Hoc Routing}

By ad hoc routing, we mean that MSs only exchange information in wireless channel with a bandwidth $W$, ignoring the effect of BSs.

Definition 9: (Scheduling Scheme $S^{*}$ ) $S^{*}$ schedules node $i$ transmit to node $j$ at time slot $t$ according to the protocol model defined in definition 5 with the transmission range $R_{T}=\frac{c_{T}}{\sqrt{n}}$, which is warrant by [11].

The next lemma is provided by [3]. It establishes a relationship between link capacity and encounter probability considering mobility.

Lemma 3: In an uniformly dense network with bandwidth $W$, under scheduling $S^{*}$, the link capacity between two nodes (at least contain one MS) is:

$$
\mu^{S^{*}}(i, j)=\Theta\left(W \cdot \operatorname{Pr}\left\{d_{i j} \leq \frac{c_{T}}{\sqrt{n}} \mid \mathbf{H}_{\mathbf{n}+\mathbf{m}}\right\}\right)
$$

Corollary 1: The link capacities between MSs $i, j$ and BS $l$ are:

$$
\begin{aligned}
\mu\left(X_{i}^{h}, X_{j}^{h}\right) & =\Theta\left(W \cdot \frac{f^{2}(n)}{n} \eta\left(f(n)\left\|X_{i}^{h}-X_{j}^{h}\right\|\right)\right) \\
\mu\left(X_{i}^{h}, Y_{l}^{h}\right) & =\Theta\left(W \cdot \frac{f^{2}(n)}{n} s\left(f(n)\left\|Y_{l}^{h}-X_{i}^{h}\right\|\right)\right)
\end{aligned}
$$

Proof: Provided in 2.1 in supplementary file.

It is easy to prove that scheduling $S^{*}$ is optimal in order sense. On one hand, $\forall R_{T}=o(1 / \sqrt{n})$ can not fully guarantee connectivity and results in a performance decline. On the other, $\forall R_{T}=\omega(1 / \sqrt{n})$ will cause too much interference and decrease the link capacity, as well. ${ }^{1}$

By mapping the network into a Generalized Random Geometric Graph $G\left(n, n_{c}, r, \mu\right)$, we can calculate the asymptotic capacity more conveniently. In $G\left(n, n_{c}, r, \mu\right)$, we use $n$ vertices to represent the home-points. For each pair of vertices $(i, j)$, we connect them with an edge of capacity $\mu\left(\left\|X_{i}^{h}-X_{j}^{h}\right\|\right)$. The following proposition provides an upper bound of multicast capacity in the network.

Proposition 1: Define that $d_{i D}^{h}$ is the length of minimum spanning tree that covers source node $i$ and the corresponding home-points of its $k$ destinations. If throughput $\lambda$ is sustainable, we have:

$$
\lambda \sum_{s} d_{s D}^{h} \leq \sum_{i j} \mu_{i j}^{h} d_{i j}^{h}
$$

1. A formal proof for the optimality of scheduling $S^{*}$ is presented in [11]. 
Proof: For any source-destination pair $(s, d)$, traffic $\lambda$ must pass through relay pathes connecting $(s, d)$ in graph $G\left(n, n_{c}, r, \mu\right)$. By the definition of minimum spanning tree and triangle inequality, all the relay pathes connecting source $s$ and destinations $D=\left\{d_{1}, d_{2}, \ldots d_{k}\right\}$ should have a total length larger than $d_{s D}^{h}$.

Fortunately, we have a classic conclusion on $d_{s D}^{h}$.

Lemma 4: In a d-dimensional cube of side length $a$, Euclidian minimum spanning tree of $n$ randomly and uniformly distributed nodes has an asymptotic length of $\tau(d) n^{\frac{d-1}{d}}$, where $\tau(d)$ only depends on $d$.

Corollary 2: $\forall i, 1 \leq i \leq n, d_{i D}^{h}=\Theta(\sqrt{k})$.

Theorem 1: The upper bound of per-node multicast capacity in uniformly dense networks by ad hoc routing is:

$$
\lambda=O\left(\frac{W}{\sqrt{k} f(n)}\right)
$$

Proof: In the first step, we try to simplify equation (6) and derive an preliminary expression of upper bound using Proposition 1. We introduce a sum: $T^{n}(d)=-\sum_{i} \sum_{j} \mu_{i j}^{n} \mathbf{1}_{d_{i j}^{h}>d}$, which represents the opposite summation of link capacities provided by links satisfying $d_{i j}^{h}>d$. By the definition, it is obvious that $T(d+\Delta)-T(d)=\sum_{i} \sum_{j} \mu_{i j}^{n} \mathbf{1}_{d<d_{i j}^{h}<d+\Delta}$, so we have :

$$
\sum_{i} \sum_{j} \mu_{i j}^{n} d_{i j}^{h}=\int x d T^{n}(x)
$$

Applying this to Proposition 1 results in:

$$
\lambda \sum_{s} d_{s D}^{h} \leq \int x d T^{n}(x)
$$

Combining with Corollary 2, we have:

$$
\lambda=O\left(\frac{1}{n \sqrt{k}} \int x d T^{n}(x)\right)
$$

In the second step, we calculate $\int x d T^{n}(x)$ to finish the proof. Let $D=\Theta(1 / f(n))$, we can divide $\int x d T^{n}(x)$ into $\int_{x \leq D} x d T^{n}(x)$ and $\int_{x>D} x d T^{n}(x)$.

On one hand, $\int_{x \leq D} x d T^{n}(x)$ is the summation of link capacities whose home-points are within distance $\Theta(1 / f(n))$. This part of integration sums up link capacities only when two nodes are close to each other. In other words, there exists a constant $y_{0}$, such that $\operatorname{Pr}\left\{d_{i j} \leq R_{T}(n)\right\}>0$ only when $\left\|X_{i}^{h}-X_{j}^{h}\right\| \leq \frac{y_{0}}{f(n)}$. Meanwhile, considering the limited bandwidth for each node, it is obvious that $\mu_{i}^{n}=\sum_{j} \mu_{i j}^{n} \leq W$. Assume that there is a simple curve $\mathcal{L}$ dividing $\mathcal{O}$ into $A_{0}$ and $B_{0}$. The information flow crossing $\mathcal{L}$ is $\sum_{i \in A_{0}} \sum_{j \in B_{0}} \mu_{i j}^{n}$. Define $C_{0}$ as the set of nodes that are within $\frac{y_{0}}{f(n)}$ away of $\mathcal{L}$. Combining with premise that $x \leq D$, we have:

$$
\begin{aligned}
\sum_{i \in A_{0}} \sum_{j \in B_{0}} \mu_{i j}^{n} & =\sum_{i \in C_{0}} \sum_{j \in B_{0}} \mu_{i j}^{n} \\
& \leq \sum_{i \in C_{0}} \mu_{i}^{n} \leq W \cdot N\left(C_{0}\right) \leq \Theta\left(\frac{W \cdot n}{f(n)}\right)
\end{aligned}
$$

Substituting this into (8), it follows:

$$
\lambda=O\left(\frac{W}{\sqrt{k} f(n)}\right)+O\left(\frac{1}{n \sqrt{k}} \int_{x>\frac{1}{f(n)}} x d T_{n}(x)\right)
$$

On the other hand, we try to find a simple lower bound $S^{n}(d)$ of $T^{n}(d)$ to calculate $\int_{x>D} x d T^{n}(x)$. The $S^{n}(d)$ should satisfy 1$) \sup ^{n}(d)=0$ and 2) $T^{n}(d) \geq$ $S^{n}(d)$. And this results in:

$$
\int_{x>D} x d T^{n}(x) \leq \int_{x>D} x d S^{n}(x)
$$

By the definition of transmission range, we have:

$$
\begin{aligned}
\operatorname{Pr}\left\{d_{i j}(t) \leq R_{T}\right\} \leq & \operatorname{Pr}\left\{\left\|X_{i}(t)-X_{i}^{h}\right\|>\frac{d_{i j}^{h}-R_{T}}{2}\right\} \\
& +\operatorname{Pr}\left\{\left\|X_{j}(t)-X_{j}^{h}\right\|>\frac{d_{i j}^{h}-R_{T}}{2}\right\} \\
= & 2 \int_{\mathcal{O}} \phi^{n}(x) \mathbf{1}_{x>\frac{d_{i j}^{h}-R_{T}}{2}} d x
\end{aligned}
$$

Considering the definition of $T(d)$, we have:

$$
\begin{aligned}
T(d) & \geq W \cdot-\sum_{i} E\left[\mathbf{1}_{\left.\left\|X_{i}(t)-X_{i}^{h}\right\|>\frac{d-R_{T}}{2}\right]}\right. \\
& \geq-2 n W \cdot \int_{\mathcal{O}} \phi^{n}(x) \mathbf{1}_{x>\frac{d_{i j}^{h}-R_{T}}{2}} d x=S(d)
\end{aligned}
$$

For any constant $M$, it follows:

$$
\begin{aligned}
& \int_{x>D} x d T^{n}(x) \\
& \leq \frac{-W}{n \sqrt{k}} \int_{y>\frac{M}{f(n)}} 2 n y \cdot \frac{d}{d y}\left(\int_{X \in \mathcal{O}} \phi^{n}(\|X\|) \xi(\|X\|) d X\right) d y
\end{aligned}
$$

where $\xi(\|X\|)=\mathbf{1}_{\|X\|>\frac{d_{i j}^{h}-R_{T}}{2}}$ We take $M=10$ (which does not affect the result in order sense), and when $n$ is large enough, for any $y \geq \frac{10}{f(n)}$, it is true that $\frac{y-R_{T}}{2}>\frac{y}{3}$. So that:

$$
\begin{aligned}
& \int_{x>D} x d T^{n}(x) \\
& \leq \frac{-W}{n \sqrt{k}} \int_{y>\frac{10}{f(n)}} 2 n y \cdot \frac{d}{d y}\left(\int_{X \in \mathcal{O}} \phi^{n}(\|X\|) \xi(\|X\|) d X\right) d y \\
& \approx \frac{2 W}{\sqrt{k}} \int_{y>\frac{10}{f(n)}} y\left[2 \pi y \phi^{n}\left(\frac{y}{3}\right)\right] d y \\
& <\frac{4 \pi W}{\sqrt{k}} \int_{y} f^{2}(n) y^{2} \phi\left(f(n)\left(\frac{y}{3}\right)\right) d y=\Theta\left(\frac{W}{\sqrt{k} f(n)}\right)
\end{aligned}
$$

Finally, we have:

$$
\lambda=O\left(\frac{W}{\sqrt{k} f(n)}\right)+O\left(\frac{W}{\sqrt{k} f(n)}\right)=O\left(\frac{W}{\sqrt{k} f(n)}\right)
$$




\subsection{Lower Bound in Uniformly Dense Networks by Ad Hoc Routing}

In the following part of this section, we will derive asymptotically reachable lower bound on multicast capacity in uniformly dense networks by ad hoc routing. We have already mapped a mobile network into a static graph, which makes the establishment of a multicast routing possible and realistic. We employ the next algorithm from [7] to set up the multicast routing in graph $G\left(n, n_{c}, r, \mu\right)$.

Algorithm 1 Optimal ad hoc routing for multicast transmission $U_{i}=\left\{s_{i}, d_{i, 1}, d_{i, 2}, \ldots, d_{i, k}\right\}$

1. Divide $\mathcal{O}$ into squarelets, of which the side length is $c / f(n)$. And we will have $f^{2}(n) / c^{2}$ such squarelets. We denote the squarelet in row $i$ column $j$ as $(i, j)$.

2. We use the following scheme to generate the Euclidian Spanning Tree $\operatorname{EST}\left(U_{i}\right)$

(1) Let the $k+1$ nodes form $k+1$ connected components;

(2) repeat (3) and (4) for $g=1,2,3, \ldots, k$;

(3) in the $g$ th step, divide $\mathcal{O}$ into at most $k-g$ square cells, each with side length $\frac{1}{\sqrt{k-g}}$;

(4) choose a cell that contain two nodes from different connected components, then connect them using Manhattan routing and merge the corresponding components.

3. For each link $u v$ in $\operatorname{EST}\left(U_{i}\right)$, assume that $u$ and $v$ are located inside squarelets $\left(i_{u}, j_{u}\right)$ and $\left(i_{v}, j_{v}\right)$. In squarelet $\left(i_{u}, j_{v}\right)$ or $\left(i_{v}, j_{u}\right)$, choose a node $w$, so that $u w v$ is the Manhattan routing connecting $u$ and $v$. Repeat this for each link in $\operatorname{EST}\left(U_{i}\right)$, and merge the corresponding link into Manhattan tree $M T\left(U_{i}\right)$.

4. For each link $u w$, find a node in each of the squarelets that are crossed by line $u w$. Obtain a path $P(u, w)$ by connecting all of this nodes. Then delete those not in $U_{i}$ and obtain the multicast tree $\operatorname{MTR}\left(U_{i}\right)$.

5. Return $\operatorname{MTR}\left(U_{i}\right)$.

Lemma 5: By Algorithm 1, the probability that a random multicast flow will be routed through a certain squarelet $A$ is at most $c^{\prime} \sqrt{k} c / f(n)$, where $c^{\prime}$ is a constant.

Proof: Provided in 2.2 in supplementary file.

Theorem 2: With the MTR generated by Algorithm 1, the sustainable per-node multicast capacity by ad hoc routing in dense networks is $\lambda=\Theta\left(\frac{1}{\sqrt{k} f(n)}\right)$.

Proof: In a random geometric graph $G$, the number of edges from squarelet $A$ to squarelet $B$ is $N(A) N(B)$. If a per-node capacity $\lambda$ is sustainable, each of these edges should not be overloaded. Considering the maximum of flows crossing one node defined by Lemma 5, we have:

$$
\begin{aligned}
\frac{\lambda \frac{c^{\prime} c n \sqrt{k}}{f(n)}}{\underline{N}(A) \underline{N}(B)} & =\Theta\left(\lambda \cdot \frac{\sqrt{k} f^{3}(n)}{n}\right) \\
& \leq W \cdot \mu^{n}\left(\bar{d}_{A, B}\right)=W \cdot g(n) \eta(\sqrt{5} c)
\end{aligned}
$$

where $\bar{d}_{A, B}=\frac{\sqrt{5} c}{f^{3}(n) \sqrt{k} / n}$.

To sum up, the sustainable per-node multicast capacity in uniformly dense networks by ad hoc routing is:

$$
\lambda=\Theta\left(\frac{W \cdot g(n) \eta(\sqrt{5} c)}{\frac{\sqrt{k} f^{3}(n)}{n}}\right)=\Theta\left(\frac{W}{\sqrt{k} f(n)}\right)
$$

\section{Multicast Capacity in UNiformLy Dense Networks by Cellular Routing}

In this section, we consider the impact of infrastructure in multicast capacity of a mobile network. Multicast flows will be routed through BSs. We divide the bandwidth in air channels into uplink bandwidth $W_{A}$ and downlink bandwidth $W_{C}$. We further assume that the bandwidth of optical fibers connecting BSs is $W_{B}$.

Definition 10: (Cellular Routing $R^{C}$ ) Cellular routing $R^{C}$ consists of three phases. In the first phase, a multicast source node routes the packets to a BS. In the second phase, the packets are routed to the cells that contain destinations. In the last phase, BSs of these cells broadcast packets to the destinations.

It is worth pointing out that such a routing cannot be established directly in mobile networks. However, with the help of the mapping scheme presented in the previous section, it is possible to generate a cellular multicast route in a random geometric graph.

\subsection{Upper Bounds in Uniformly Dense Networks by Cellular Routing}

Since cellular routing $R^{C}$ is divided into three phases, the capacity of cellular routing $R^{C}$ is restricted by the worst case among three phases. We firstly explore the upper bound in each phase, then combine them together to obtain the overall upper bound.

\subsubsection{Upper Bound in Phase 1}

In phase $1, n$ MSs act as sources, and try to forward their messages to one of the BSs. Scheduling policy $S^{*}$ is applied in this phase, and the uplink bandwidth for each BS is $W_{A}$.

Lemma 6: The upper bound of per-node capacity in uniformly dense networks with $m$ BSs and $n$ MSs, in phase 1 of cellular routing $R^{C}$, is :

$$
\lambda_{p 1}=O\left(\frac{m W_{A}}{n}\right)
$$

Proof: Because of the limited antennas, a BS can serve at most $\Theta(1)$ MSs in each time slot. That means one BS can at most receive $\Theta\left(W_{A}\right)$ traffic in each time slot. By applying a simple TDMA or FDMA scheme, the maximum of total available uplink resource is $\Theta\left(W_{A} \cdot m\right)$. All the $n$ MSs share this resource equally, which means that per-node capacity of each MS can not exceed $\Theta\left(\frac{m W_{A}}{n}\right)$. 


\subsubsection{Upper Bound in Phase 2}

In phase 2, BSs exchange messages received from MSs through optical fibers, each of which has a bandwidth of $W_{B}$. We map the network only consisting BSs into a random geometric graph $G_{B}\left(m, W_{B}\right) . m$ vertices are used to represent the BSs. The capacities of edges connecting each pair of BSs $(i, j)$ are $W_{B}$. The next proposition holds in such graph.

Definition 11: (Inner and outer tessellation) Consider a torus, which is regularly divided into multiple squarelets. Assume that, in this torus, there is a subset $\Psi$ bounded by a closed and convex boundary $\mathcal{L}$. The inner tessellation of this torus, denoting as $\underline{\Psi}$, contains squarelets strictly within $\Psi$. And the outer tessellation is a union of $\underline{\Psi}$ and all the squarelets crossed by boundary $\mathcal{L}$, denoting as $\bar{\Psi}$.

Proposition 2: In a random geometric graph, traffic $\lambda$ is sustainable only if, for any partition $(S, D)$ of vertices, it holds:

$$
\lambda \sum_{s \in S} \sum_{d \in D} \lambda_{s d} \leq \sum_{i \in S} \sum_{j \in D} \mu_{i j}^{n}
$$

Lemma 7: The upper bound of per-node capacity in uniformly dense networks with $m$ BSs and $n$ MSs, in phase 2 of cellular routing $R^{C}$, is :

$$
\lambda_{p 2}=O\left(\frac{m^{2} W_{B}}{k n}\right)
$$

Proof: Provided in 2.3 in supplementary file.

\subsubsection{Upper Bound in Phase 3}

In phase 3, each BS transmits messages in its own cell. Optimal scheduling policy $S^{*}$ is applied in this phase again, and the downlink bandwidth for each BS is $W_{C}$. We map the network consisting of $m$ BSs and $n$ MSs into a random geometric graph $G_{C}\left(m+n, W_{C}\right)$. $m$ vertices are used to represent the BSs, and the other $n$ are for MSs. Edges only exist from BSs to MSs, with capacity $\mu\left(Y_{i}^{h}, X_{j}^{h}\right)$. There is no link among BSs, as well as MSs.

Proposition 3: Given an arbitrary non-increasing function $\mathrm{s}(\mathrm{x})$ with finite support and $X \in \mathcal{O}$, it holds:

$$
\int_{Y \in \mathcal{O}} s(f(n)\|Y-X\|) d Y \sim \frac{1}{f^{2}(n)}
$$

Lemma 8: The upper bound of per-node capacity in uniformly dense networks with $m$ BSs and $n$ MSs, in phase 3 of cellular routing $R^{C}$, is :

$$
\lambda_{p 3}=O\left(\frac{m W_{C}}{k n}\right)
$$

Proof: Provided in 2.4 in supplementary file.

As previously described, cellular routing $R^{C}$ is a serial connection of the above 3 phases. According to its characteristic, the overall capacity can not exceed the capacity of any element in the serial connection. With Lemma $6-8$, we have the following theorem.
Theorem 3: The upper bound of per-node multicast capacity in uniformly dense networks by cellular routing $R^{C}$ is:

$$
O\left(\min \left[\frac{m W_{A}}{n}, \frac{m^{2} W_{B}}{k n}, \frac{m W_{C}}{k n}\right]\right)
$$

\subsection{Lower Bounds in Uniformly Dense Networks by cellular routing $R^{C}$}

Similar to the derivation of upper bounds by cellular routing $R^{C}$, we derive lower bounds of cellular routing $R^{C}$ in 3 phases, respectively. Then a combination of the lower bounds is presented.

\subsubsection{Lower Bound in Phase 1}

Lemma 9: A traffic rate $\Theta\left(\frac{m W_{A}}{n}\right)$ is sustainable from any MS to infrastructure system in phase 1 of cellular routing $R^{C}$.

Proof: Provided in 2.5 in supplementary file.

\subsubsection{Lower Bound in Phase 2}

Lemma 10: A traffic rate $\Theta\left(\frac{m^{2} W_{B}}{k n}\right)$ is sustainable between BSs in phase 2 of cellular routing $R^{C}$.

Proof: Provided in 2.6 in supplementary file.

\subsubsection{Lower Bound in Phase 3}

Let $f_{i}$ denote the number of multicast flows, each of which has as least one destination inside $i$ th BS's cell (or transmission range). We borrow the next lemma from [10] to facilitate our proof.

Lemma 11: When $\max \left(\frac{32 m \log m}{k} \log \frac{52 m}{k}, \frac{16 m}{k} \log (2 n)\right)$ is satisfied, the variable $\max _{j}^{m} f_{j}$ is $\Theta\left(\frac{n_{s} k}{m}\right)$ w.h.p., where $n_{s}$ is the number of source nodes.

Lemma 12: A traffic rate $\Theta\left(\frac{m W_{C}}{k n}\right)$ is sustainable from one BS to MSs in phase 3 of cellular routing $R^{C}$.

Proof: Provided in 2.7 in supplementary file

An overall lower bound of cellular routing $R^{C}$ can be obtained by combining the lower bounds in 3 phases. With Lemma 9,10,12, we can draw the following conclusion:

Theorem 4: The lower bound of per-node multicast capacity in uniformly dense networks by cellular routing $R^{C}$ is:

$$
\Theta\left(\min \left[\frac{m W_{A}}{n}, \frac{m^{2} W_{B}}{k n}, \frac{m W_{C}}{k n}\right]\right)
$$

\section{Multicast Capacity in Uniformly Dense Networks by Hybrid Routing}

At the beginning of this section, we analyze the upper bound of multicast capacity for arbitrary hybrid routing. Then reachable lower bound and corresponding routing scheme are presented. The hybrid routing utilizes both ad hoc routing and cellular routing, with the purpose of further improving the network capacity and system throughput. 


\subsection{Upper and Lower Bounds by Hybrid Routing}

Theorem 5: In uniformly dense networks, the upper bound of per-node multicast capacity by any hybrid routing is:

$$
O\left\{\max \left[\frac{W}{\sqrt{k} f(n)}, \min \left(\frac{m W_{A}}{n}, \frac{m^{2} W_{B}}{k n}, \frac{m W_{C}}{k n}\right)\right]\right\}
$$

Proof: Combining Theorem 1 and 3 , it is obvious that, in uniformly dense networks, per-node multicast capacity $\lambda$ is upper-bounded by:

$$
\lambda=O\left(\frac{W}{\sqrt{k} f(n)}\right)+O\left[\min \left(\frac{m W_{A}}{n}, \frac{m^{2} W_{B}}{k n}, \frac{m W_{C}}{k n}\right)\right]
$$

By the limit theory, we can rewrite the above expression as:

$$
\lambda=O\left\{\max \left[\frac{W}{\sqrt{k} f(n)}, \min \left(\frac{m W_{A}}{n}, \frac{m^{2} W_{B}}{k n}, \frac{m W_{C}}{k n}\right)\right]\right\}
$$

Definition 12: (Hybrid Routing Scheme $R^{H}$ ) Hybrid Routing Scheme $R^{H}$ evaluates both pure ad hoc routing and cellular routing $R^{C}$, and adaptively selects a better scheme, which provides larger throughput, to route the packets.

Applying Theorem 2 and 4 together, the following theorem holds beyond doubt.

Theorem 6: By hybrid routing $R^{H}$, the sustainable pernode multicast throughput in uniformly dense networks is:

$$
\Theta\left\{\max \left[\frac{W}{\sqrt{k} f(n)}, \min \left(\frac{m W_{A}}{n}, \frac{m^{2} W_{B}}{k n}, \frac{m W_{C}}{k n}\right)\right]\right\}
$$

Theorem 6 shows that our hybrid routing scheme $R^{H}$ is optimal in order sense. Moreover, it proves that the upper bound purposed by Theorem 5 is tight.

\subsection{Discussion on Capacity Regimes}

Proposition 4: The optimal frequency allocation between $W_{A}$ and $W_{C}$ is:

$$
W_{A}=\frac{W}{k+1}, \quad W_{C}=\frac{k W}{k+1}
$$

Proof: The optimal frequency allocation focuses on maximizing the uplink and downlink capacity between MSs and BSs. According to Theorem 6, the capacity $\lambda_{U D}$ that can be provided by uplink and downlink transmissions is $\lambda_{U D}=\Theta\left[\min \left(\frac{m W_{A}}{n}, \frac{m W_{C}}{k n}\right)\right]$. If $\frac{m W_{A}}{n}>\frac{m W_{C}}{k n}$, we have

$$
\lambda_{U D}=\frac{m W_{C}}{k n}<\frac{m W}{(k+1) n}
$$

If $\frac{m W_{A}}{n}<\frac{m W_{C}}{k n}$, we have

$$
\lambda_{U D}=\frac{m W_{A}}{n}<\frac{m W}{(k+1) n}
$$

However, if we allocate $W$ as $W_{A}=\frac{W}{k+1}, W_{C}=\frac{k W}{k+1}$, it results:

$$
\lambda_{U D}=\frac{m W}{(k+1) n}
$$

The multicast capacity in uniformly dense networks by hybrid routing can be represented by the following parameters: $m=\Theta\left(n^{M}\right), k=\Theta\left(n^{K}\right), W_{B}=\Theta(W$. $n^{\varphi}$ ) and $f(n)=n^{\alpha}$. If $\varphi>-M$, the bottleneck of cellular routing falls in the wireless access. Otherwise, the backbone transmission becomes the limitation. Graphical illustration of capacity regimes is provided in 1.2 in supplementary file.

\section{Multicast Capacity in NON-UniformLy Dense Networks}

In the previous sections, we discuss the multicast capacity of mobile uniformly dense networks, where $f(n) \sqrt{\gamma(n)}=o(1)$. Now we will present the results in mobile non-uniformly dense networks.

In non-uniformly dense networks without the support of BSs, a larger transmission range should be adopted to guarantee connectivity. In [3], it is proved that this transmission range should be $R_{T}=\Omega(1 / \sqrt{n})$, which only provides a capacity of $\Theta\left(\frac{n_{c}}{n^{2} \log n_{c}}\right)$. The poor capacity is a consequence of more interferences brought by larger transmission range. Considering this, we propose to decrease the transmission range of nodes, and employ BSs to guarantee connectivity. A useful lemma is cited from [11], so as to facilitate our proof.

Lemma 13: Nodes with transmission range $R_{T}=$ $r \sqrt{\frac{n_{c}}{n}}$ will cause no interference to those in other clusters, with high probability.

According to the new transmission range, we propose a new scheduling scheme.

Definition 13: (Scheduling Scheme $\widetilde{S}$ ) $\widetilde{S}$ schedules node $i$ transmit to node $j$ at time slot $t$, the transmission subjects to the restriction in definition 5 with the transmission range $R_{T}=c_{T} r \sqrt{\frac{n_{c}}{n}}$.

\subsection{Multicast Capacity in Weak Mobility Regime}

Under scheduling scheme $\widetilde{S}$, MSs in different clusters cannot communicate with each other directly in air channels. A hybrid routing is proposed to finish the transmission.

Definition 14: (Hybrid Routing Scheme $\widetilde{R}$ ) Hybrid Routing Scheme $\widetilde{R}$ consists of 2 phases. In phase 1, each source node transmits packets to destinations in its own cluster. In phase 2, each source node employs cellular routing (as defined in Definition 10) to transmit packets to destinations in other clusters.

An example of hybrid routing scheme $\widetilde{S}$ in weak mobility regime is illustrated in 1.3 in supplementary file. 


\subsubsection{Multicast Capacity in Phase 1 of $\widetilde{R}$}

Since there is no inter-cluster interference in phase 1 , each cluster acts like a sub-network independently. Next, we will prove that these sub-networks are uniformly dense in weak mobility regime, and extend the results in uniformly dense networks into this phase.

Theorem 7: In weak mobility regime, each cluster forms an independent sub-network, which is uniformly dense.

Proof: By Lemma 13, it is obvious that ad hoc transmissions within one cluster are independent of those in other clusters, since the transmission range is less than the interference range.

It is crucial to determine the key parameters $\left(\tilde{f}(n), \tilde{n}, \tilde{m}, \tilde{k}, \tilde{n}_{c}, \tilde{R}_{T}\right)$ of an arbitrary sub-network, if we want to prove it to be uniformly dense. A direct application of Chernoff bound results in the following expressions. For $\forall \varepsilon>0$, these hold w.h.p.:

$$
\begin{aligned}
& \frac{(1-\varepsilon) n}{n_{c}} \quad<\tilde{n}<\frac{(1+\varepsilon) n}{n_{c}} \\
& \frac{(1-\varepsilon) m}{n_{c}} \quad<\tilde{m}<\frac{(1+\varepsilon) m}{n_{c}} \\
& \frac{(1-\varepsilon) k}{n_{c}} \quad<\tilde{k}<\frac{(1+\varepsilon) k}{n_{c}}
\end{aligned}
$$

The definition of $\widetilde{S}$ already provides that $\widetilde{R}_{T}=$ $\left(c_{T} \cdot r \sqrt{\frac{n_{c}}{n}}\right) / r=c_{T} \sqrt{\frac{n_{c}}{n}}$. Also, by the definition of the cluster model, home-points in each cluster are randomly, independently and uniformly placed, so we have $\tilde{n}_{c}=\tilde{n}$. Since that each cluster has a radius of $r=\Theta\left(n^{-R}\right)$, the radius of each sub-network is $r+D / f(n)$, where $\mathrm{D}$ is a constant such that $D=\sup \{d \mid s(d)>0\}$.

Now we can normalize the area of each sub-network into 1. Correspondingly, we have $\tilde{f}(n)=\frac{1}{r+D / f(n)} \sim n^{R}$. According to the definition of weak mobility, we have $f(n) \sqrt{\widetilde{\gamma}(n)}=o(1)$, which can be extended as:

$$
\begin{aligned}
f(n) \sqrt{\tilde{\gamma}(n)} & =f(n) \cdot \frac{1}{r} \sqrt{\frac{\log \left(n / n_{c}\right)}{n / n_{c}}} \\
& \sim \tilde{f}(n) \sqrt{\frac{\log \tilde{n}}{\tilde{n}}}=\tilde{f}(n) \sqrt{\frac{\log \tilde{n}_{c}}{\tilde{n_{c}}}}
\end{aligned}
$$

It yields:

$$
\tilde{f}(n) \sqrt{\frac{\log \tilde{n}_{c}}{\tilde{n_{c}}}}=o(1)
$$

Applying Lemma 2 in all sub-networks guarantees that each of them is uniformly dense.

With Theorem 7, we can map our results in Section III directly into phase 1 of $\widetilde{R}$, and further obtain the following theorem.

Theorem 8: The per-node multicast capacity of phase 1 in $\widetilde{R}$ is

$$
\widetilde{\lambda}_{p 1}=\Theta\left(\frac{W \sqrt{n_{c}}}{n^{R} \sqrt{k}}\right)
$$

\subsubsection{Multicast Capacity in Phase 2 of $\widetilde{R}$}

Since we apply cellular routing $R^{C}$ in phase 2 hybrid routing scheme $\widetilde{R}$, we can further divide phase 2 of $\widetilde{R}$ into three serial steps. In the first step, source nodes deliver their packets to BSs in the same cluster, through wireless channels with uplink bandwidth $W_{A}$. Then, with the help of infrastructure, each packet is forwarded to the clusters, where the destinations locate. The traffic rate between BSs is $W_{B}$. In the last step, BSs in each cluster transmit the messages to the destinations in wireless channels with downlink bandwidth $W_{\widetilde{R}}$. If we can determine the parameters in phase 2 of $\widetilde{R}$ similar to those in cellular routing $R^{C}$, previous results can be mapped into this phase of $\widetilde{R}$.

In the first step, transmissions in different clusters cause no interference to each other, which is guaranteed by Theorem 7. Considering a sub-network formed by an arbitrary cluster, there are $\tilde{n}=\Theta\left(n / n_{c}\right)$ source nodes try to transmit their packets to $\tilde{m}=\Theta\left(m / n_{c}\right)$ BSs. Consequently, a direct mapping of Lemma 6 and 9 ensures the following assertion:

Lemma 14: The per-node capacity in the first step of $\widetilde{R}^{\prime}$ s phase 2 is:

$$
\Theta\left(\frac{\tilde{m} W_{A}}{\tilde{n}}\right)=\Theta\left(\frac{m W_{A}}{n}\right)
$$

In the second step, exchanges of information take place in the infrastructure system. The transmissions involve $m$ BSs and $n$ MSs, which is the same as phase 2 in $R^{C}$. The only difference is the number of destination nodes in other clusters, which is $k-\tilde{k}$ in this step. Therefore, Lemma 7 and 10 can be applied in this step with the above parameters, and result in the next lemma:

Lemma 15: The per-node capacity in the second step of $\widetilde{R}^{\prime}$ s phase 2 is:

$$
\Theta\left(\frac{m^{2} W_{B}}{(k-\tilde{k}) n}\right)=\Theta\left(\frac{n_{c} m^{2} W_{B}}{\left(n_{c}-1\right) n k}\right)
$$

In the last step, BSs transmit packets to all the destinations in the same cluster. As previously described, there are $\tilde{m}$ BSs and $\tilde{n}$ MSs in each cluster. The number of destination nodes in each cluster is $k^{(d e s)}=\frac{(k-\tilde{k})}{n_{c}}=$ $\Theta\left(\frac{k}{n_{c}}-\frac{k}{n_{c}^{2}}\right)=\Theta\left(\frac{k}{n_{c}}\right)$, which can be proved by utilizing Lemma 1. Substituting the above parameters into Lemma 8 and 12, the following lemma holds in this step.

Lemma 16: The per-node capacity in the last step of $\widetilde{R}^{\prime}$ s phase 2 is:

$$
\Theta\left(\frac{\tilde{m} W_{C}}{k^{(d e s)} \tilde{n}}\right)=\Theta\left(\frac{m n_{c} W_{C}}{k n}\right)
$$

Now that we have the results in all the serial components of hybrid routing $\widetilde{R}$, the overall capacity should be restricted by the minimum throughput of all the components. As a result, we provide the following theorem, which holds w.h.p. in weak mobility regime. 
Theorem 9: In weak mobility regime, the per-node multicast capacity of mobile ad hoc networks with infrastructure support, by hybrid routing scheme $\widetilde{R}$, is:

$$
\Theta\left[\min \left(\frac{W \sqrt{n_{c}}}{n^{R} \sqrt{k}}, \frac{m W_{A}}{n}, \frac{n_{c} m^{2} W_{B}}{\left(n_{c}-1\right) n k}, \frac{m n_{c} W_{C}}{n k}\right)\right]
$$

\subsection{Multicast Capacity in Trivial Mobility Regime}

Applying the connectivity criterion in [17], the critical transmission range within each cluster is $R_{T}^{\prime}=$ $r \sqrt{\log (\tilde{n}) / \pi \tilde{n}}$ when $f(n) \sqrt{\tilde{\gamma}(n)}=\omega(1)$. From Lemma 13 , it is obvious that $R_{T}^{\prime}$ will cause no inter-cluster interference. We can map each cluster into sub-network almost the same as Theorem 7 . The only difference is that $\widetilde{R}_{T}=\sqrt{\log (\tilde{n}) / \pi \tilde{n}}$.

Theorem 10: In trivial mobility regime, nodes' mobility are negligible and the whole network acts as a static one.

Proof: The proof is shown in 2.7 in supplementary file.

With the help of Theorem 8, we can extend existing studies of static networks into trivial mobility regime of mobile networks. Since pervious studies, such as [4], [9], [10], [11], etc., have already discussed capacity of static networks with infrastructure support, one can refer to theses works for more details.

\section{Discussion}

We use algorithm 2 to illustrate the proposed hybrid routing strategy.

\footnotetext{
Algorithm 2 Adaptive hybrid routing strategy $\mathcal{T}$

1. According to the network model and scaling law, we first set the value of network parameters, such as $f(n)$, $\gamma(n)$ and $\tilde{\gamma}(n)$.
}

2. We determine the mobility regime by the values of $f(n) \sqrt{\gamma(n)}$ and $f(n) \sqrt{\tilde{\gamma}(n)}$.

3. In strong mobility regime, we suggest the multicast protocol to adaptively choose ad hoc routing or cellular routing for larger throughput. In weak mobility regime, ad hoc routing is only used in intra-cluster MS-MS transmissions while cellular routing serves for inter-cluster transmissions. In trivial mobility regime, the mobility is considered to be trivial and provides little help for delivering packets, routing strategy in static networks can be adopted.

4. Return routing strategy $\mathcal{T}$.

It is worth pointing out that the mobility improves network performance in strong and weak mobility regimes. When the mobility is strong, both ad hoc routing and cellular routing are available to achieve multicast scenario. We utilize an adaptive scheme to takes the advantage of mobility and infrastructure support while circumvents the bottleneck of each of these two factors. In weak mobility regime, serial connection scheme is adopted here, and the multicast capacity subjects to the bottlenecks of wireless access and backbone transmission.

\section{Conclusion}

This paper analyzes the multicast capacity in mobile ad hoc networks with infrastructure support. Hybrid routing schemes are proposed to achieve reachable upper and lower bounds in each of the regimes. It is worth pointing out that our work generalizes results in previous works on hybrid networks, impact of mobility and multicast transmissions, as well as any combinations of the above. Our results are instructive in the design of real hybrid system combining cellular and ad hoc networks.

\section{ACKNOWLEDGMENT}

This paper is supported by National Fundamental Research Grant (No. 2011CB302701); NSF China (No. 61271219,61202373); Shanghai Basic Research Key Project (No. 11JC1405100); China Ministry of Education Fok Ying Tung Fund (No. 122002); China Ministry of Education New Century Excellent Talent (No. NCET-10-0580); China Postdoctoral Science Foundation Grant 2011M500774 and 2012T50417, STCSM Grant 12JC1405200 and SEU SKL project \#2012D13.

\section{References}

[1] P. Gupta and P. R. Kumar, "The capacity of wireless networks," IEEE Trans. Inf. Theory, vol. 46, no. 2, pp. 388-404, 2000.

[2] M. Grossglauser and D. N. C. Tse, "Mobility increases the capacity of ad hoc wireless networks," IEEE/ACM Trans. Networking, vol. 10, no. 4, pp. 477-486, 2002.

[3] M. Garetto, P. Giaccone, and E. Leonardi, "Capacity scaling in delay tolerant networks with heterogeneous mobile nodes," in ACM MobiHoc 2007, New York, USA, 2007, pp. 41-50.

[4] B. Liu, Z. Liu, and D. Towsley, "On the capacity of hybrid wireless networks," in IEEE Infocom 2003, vol. 2, San Francisco, USA, 2003, pp. 1543-1552.

[5] U. C. Kozat and L. Tassiulas, "Throughput capacity of random ad hoc networks with infrastructure support," in ACM MobiCom 2003, New York, USA, 2003, pp. 55-65.

[6] A. Agarwal and P. R. Kumar, "Capacity bounds for ad hoc and hybrid wireless networks," SIGCOMM Comput. Commun. Rev., vol. 34, no. 3, pp. 71-81, 2004.

[7] X.-Y. Li, "Multicast capacity of wireless ad hoc networks," IEEE/ACM Trans. Networking, vol. 17, no. 3, pp. 950-961, 2009.

[8] X.-Y. Li, Y. Liu, S. Li, and S. Tang, "Multicast capacity of wireless ad hoc networks under gaussian channel model," IEEE/ACM Trans. Networking, vol. 18, no. 4, pp. 1145-1157, 2010.

[9] X. Mao, X.-Y. Li, and S. Tang, "Multicast capacity for hybrid wireless networks," in ACM MobiHoc 2008, Hong Kong, China, 2008, pp. 189-198.

[10] X.-Y. Li, X. Mao, and S. Tang, "Closing the gap of multicast capacity for hybrid wireless networks," 2009, [Online]. Available: http:/ / www.cs.iit.edu/ xli.

[11] W. Huang, X. Wang, and Q. Zhang, "Capacity scaling in mobile wireless ad hoc network with infrastructure support," in IEEE ICDCS, 2010, Genoa, Italy, 2010, pp. 848-857.

[12] M. J. Neely and E. Modiano, "Capacity and delay tradeoff for ad hoc mobile networks," IEEE Trans. Inf. Theory, vol. 51, no. 6, pp. 1917-1937, 2005.

[13] Y. Guo, F. Hong, Z, Jin, Y. He, Y. Feng and Y. Liu, "Perpendicular Intersection: Locating Wireless Sensors with Mobile Beacon," IEEE Trans. Vehicular Technology, vol. 59, no. 7, pp. 3501-3509, 2010.

[14] C. Wang, X.-Y. Li, S. Tang, C. Jiang and Y. Liu "Capacity and delay in mobile ad hoc networks under Gaussian channel model," SIGMOBILE Mob. Comput. Commun. Rev., vol. 14, no. 3, pp. 22-24, 2010. 
[15] X. Lin, G. Sharma, R. R. Mazumdar, and N. B. Shroff, "Degenerate delay-capacity tradeoffs in ad-hoc networks with brownian mobility," IEEE/ACM Trans. Networking, vol. 14, no. SI, pp. 2777-2784, 2006.

[16] M. Garetto, P. Giaccone, and E. Leonardi, "Capacity scaling in ad hoc networks with heterogeneous mobile nodes: The supercritical regime," IEEE/ACM Trans. Networking, vol. 17, no. 5, pp. 1522-1535, 2009.

[17] P. Gupta and P. R. Kumar, "Critical power for asymptotic connectivity," in IEEE Conference on Decision and Control, 1998, vol. 1, Florida, USA, 1998, pp. 1106-1110.

[18] X. Chen, W. Huang, X. Wang, X. Lin, Multicast Capacity in Mobile Wireless Ad Hoc Network with Infrastructure Support, in Proc. of IEEE INFOCOM 2012, March, Orlando, 2012.

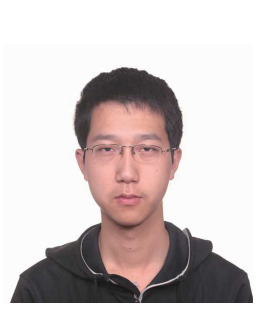

Zhenzhi Qian is currently currently pursuing the B.E. degree in electronic engineering in Shanghai Jiao Tong University, China. His research interests are in the area of asymptotic analysis of coverage and capacity in wireless ad hoc networks.

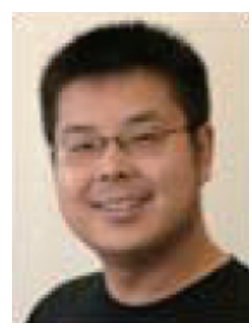

Xiaohua Tian received his B.E. and M.E. degrees in communication engineering from Northwestern Polytechnical University, Xi'an, China, in 2003 and 2006, respectively. He received the Ph.D. degree in the Department of Electrical and Computer Engineering (ECE), Illinois Institute of Technology (IIT), Chicago, in Dec. 2010. He is currently a postdoc in Department of Electronic Engineering in Shanghai Jiao Tong University, China. His research interests include wireless networking, Internet of Things and nextgeneration Internet. He serves as the guest editor of International Journal of Sensor Networks, publicity co-chair of the 7th International Conference on Wireless Algorithms, Systems, and Applications (WASA 2012). He also serves as the Technical Program Committee member for IEEE GLOBECOM 2011-2013, IEEE ICC 2013 and WASA 2011.

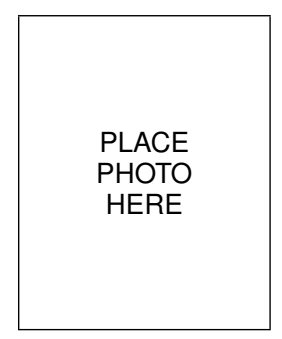

Xi Chen received the M.S. degree in electronic engineering in Shanghai Jiao Tong University, China, in 2012. His research interests are scaling law and capacity of wireless networks.

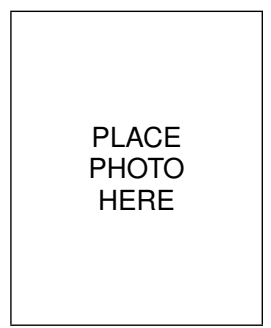

Wentao Huang received the B.S. degree in communications engineering from the Nanjing University of Posts and Telecommunications, Nanjing, China, in 2008, and received the M.S. degree in electronic engineering at Shanghai Jiao Tong University, Shanghai, China, in 2011. His current research interests include distributed systems, mobile computing, and network security.

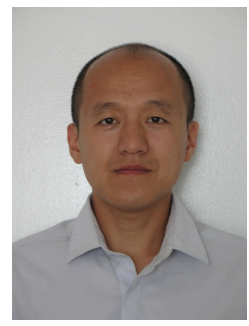

Xinbing Wang (SM'12) received the B.S. degree (with hons.) in automation from Shanghai Jiao Tong University, Shanghai, China, in 1998, the M.S. degree in computer science and technology from Tsinghua University, Beijing, China, in 2001, and the Ph.D. degree with a major in electrical and computer engineering and minor in mathematics from North Carolina State University, Raleigh, in 2006. Currently, he is a professor with the Department of Electronic Engineering, Shanghai Jiao Tong University. His research interests include resource allocation and management in mobile and wireless networks, TCP asymptotics analysis, wireless capacity, cross-layer call admission control, asymptotics analysis of hybrid systems, and congestion control over wireless ad hoc and sensor networks. Dr. Wang has been a member of the Technical Program Committees of several conferences including ACM MobiCom 2012, ACM MobiHoc 2012, 2013, IEEE INFOCOM 2009-2013. 